\title{
On the Determination of Rock Anisotropy for Stress Measurements
}

\author{
P.M. Dight Australian Centre for Geomechanics, The University of Western Australia, Australia
}

A.V. Dyskin School of Civil and Resource Engineering, The University of Western Australia, Australia

\begin{abstract}
The traditional methods of in situ stress determination can be seriously affected by rock anisotropy, which necessitates both development of the corresponding interpretation methods and accurate determination of the elastic characteristics of the anisotropic rocks. In this contribution we consider the method of determination of anisotropic moduli by testing small subcores drilled in different directions. Rock anisotropy is often induced by the presence of joints, foliation, schistosity, bedding or similar features. In the case when the spacing between the theses features exceeds the length of the subcores the results of moduli determination in separate subcores shows considerable variability. A mechanism of this variability lies in the fact that a particular subcore may or may not be intersected by a joint. We show that despite this, the averaging procedure used in the method of moduli reconstruction, developed previously by the authors, can still be successfully applied, since the averaging over the subcores recovers the large-scale moduli. However, the standard deviation associated with the randomness of joint-subcore intersection can in some case be very large (potentially unlimited) which explains the observed variability.
\end{abstract}

\section{Introduction}

Adequate determination of the stress field is an important aspect of safe mine design. Stresses in and around mineralised systems are influenced by local and regional tectonics and the rock fracture processes associated with the tectonics. The presence of these factors makes the prediction of in situ stresses quite difficult and requires several measurements/or in multiple locations.

The most used class of stress measurement techniques is overcoring which is based on the determination of local rock strain induced by stress relief caused by overcoring (if there is underground access); the stress is then recovered assuming local elastic behaviour of rock. This principle underlines such popular methods as the hollow inclusion cell (HI), door stopper and ANZI cell techniques. A key element in these methods until recently has been the assumption that the rock is isotropic. In many cases the rock anisotropy is too strong to be ignored and hence the necessary correction has to be introduced. Other stress measurement methods such as the rock memory methods, the deformation rate analysis (DRA) and acoustic emission (AE), sensor the stress by comparing with artificially induced stresses without the need to infer them from strain and are insensitive to the deformation behaviour of rock (Goodman, 1963; Kurita and Fujii, 1979; Lavrov, 2002). These methods are more sophisticated and have yet to receive the recognition they deserve. Even if the rock memory techniques are used, the general tendency is to verify their results against the results of measurements performed by traditional stress relief methods. As confidence builds, this will become less necessary. An intrinsic problem of such a comparison is the assumption of rock isotropy in the interpretation of stress relief measurement results. Such an assumption can lead to considerable deviation of the interpretation of the results from the actual stresses (Borsetto et al., 1984; Hakala et al., 2007), which invariably lead to unjustified rejection of rock memory methods. This circumstance necessitates the development of methods of correction of stress relief techniques for rock anisotropy.

Currently, the research is concentrated on the development of the methods of stress reconstruction for given anisotropic rock moduli (Amadei and Goodman, 1982; Amadei, 1983; Borsetto et al., 1984; Hakala et al., 2007), which presents a considerable challenge because analytical solutions for the general case of rock anisotropy are not available. A less recognised challenge is the determination of the moduli of anisotropic rocks. The obvious difficulty is the sheer number of samples to be tested as in the simplest case of transversal isotropic rock five moduli and two angles of orientation of the plane of isotropy have to be reconstructed. A typical way of dealing with this is sub-sampling the core in different directions. This method is capable of providing the number of measurements sufficient for reconstruction of the moduli; 
however, it is met with another challenge related to the fact that often the rock anisotropy is induced by features (e.g. joints or fractures) with spacings that can be larger than the dimensions of the sub-samples, Figure 1. In this case the sub-sample tested does not necessarily contain the modulus-affecting feature and hence gives the values of moduli not representative of the rock at the scale of stress measurements. This paper investigates this problem.

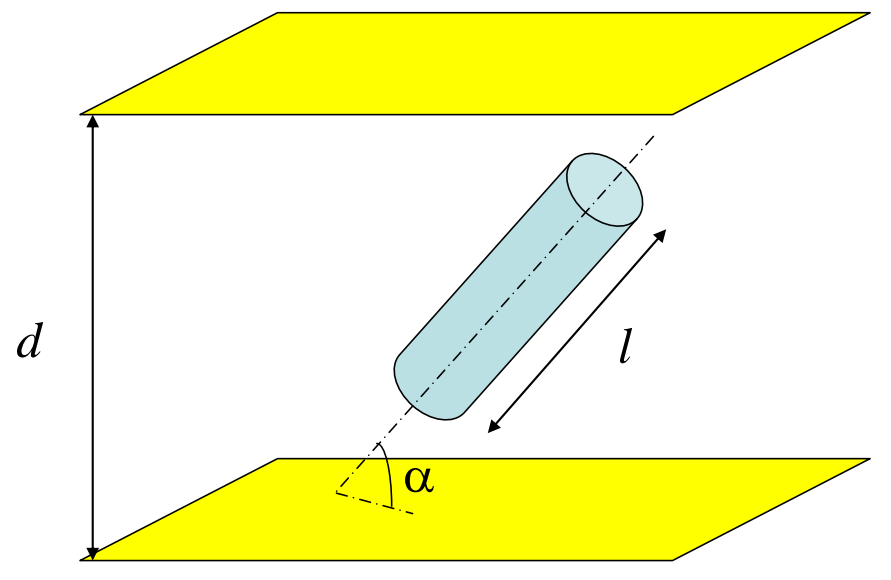

Figure 1 Position of the sub-sample and joints/foliation when the sub-sample is no longer representative of large-scale anisotropic rock. Here $d$ is the spacing between joints, $\alpha$ is the angle (to the joints) at which the subcore is cut, $l$ is the subcore length

\section{Moduli reconstruction}

\subsection{Method of moduli reconstruction from subcore testing}

In this section, the algorithm described by Dight and Dyskin (2007) is followed. Suppose we have the results of testing $N$ subcores. We relate a subcore number $n$ to a local Cartesian coordinate set $\left(x_{1}{ }^{(n)}, x_{2}{ }^{(n)}, x_{3}{ }^{(n)}\right)$ whose $x_{3}{ }^{(n)}$-axis is directed along the axis of the subcore. The results of the laboratory tests in uniaxial compressive loading of subcores are interpreted as average axial and lateral strains measured in response to the axial load as the subcore orientation with respect to its axis was not marked. Subsequently, only two average compliances, $S_{\text {axial }}^{(n)}, \quad S_{\text {lateral }}^{(n)}$ can be determined.

We now relate these measurements to a certain laboratory coordinate frame, $\left(x_{1}, x_{2}, x_{3}\right)$ corresponding to which the rock moduli are reconstructed. We use a six-dimensional matrix notation (Lekhnitskii, 1977):

$$
\left(\begin{array}{l}
\varepsilon_{11} \\
\varepsilon_{22} \\
\varepsilon_{33} \\
\varepsilon_{23} \\
\varepsilon_{13} \\
\varepsilon_{12}
\end{array}\right)=\left(\begin{array}{llllll}
S_{11} & S_{12} & S_{13} & S_{14} & S_{15} & S_{16} \\
S_{12} & S_{22} & S_{23} & S_{24} & S_{25} & S_{26} \\
S_{13} & S_{23} & S_{33} & S_{34} & S_{35} & S_{36} \\
S_{14} & S_{24} & S_{34} & S_{44} & S_{45} & S_{46} \\
S_{15} & S_{25} & S_{35} & S_{45} & S_{55} & S_{56} \\
S_{16} & S_{26} & S_{36} & S_{46} & S_{56} & S_{66}
\end{array}\right)\left(\begin{array}{l}
\sigma_{11} \\
\sigma_{22} \\
\sigma_{33} \\
\sigma_{23} \\
\sigma_{13} \\
\sigma_{12}
\end{array}\right), \quad \text { or } \quad \boldsymbol{\varepsilon}=\mathbf{S \sigma}
$$

In order to accommodate the measurements on subcores drilled in different directions, we need to express the compliances in these orientations. In a conventional manner we specify the coordinate rotation by a $3 \times 3$ rotation matrix $\mathbf{R}$. The first row of the rotation matrix consists of the projections of a unit vector directed along the $x_{1}$-axis of the rotated coordinate set with respect to the original coordinate set; the second and the third rows of the matrix result from the projections of a unit vector directed along the $x_{2}$ and $x_{3}$-axes, respectively. 
We express the general rotation as a superposition of (generally) three rotations with respect to $x_{1}, x_{2}$ and $x_{3^{-}}$ axes (Figure 2).

$$
\mathbf{S}^{\prime}=\mathbf{Q S} \mathbf{Q}^{T}
$$

$$
\mathbf{Q}=\left(\begin{array}{cccccc}
R_{11}{ }^{2} & R_{12}{ }^{2} & R_{13}{ }^{2} & R_{12} R_{13} & R_{13} R_{11} & R_{12} R_{11} \\
R_{21}{ }^{2} & R_{22}{ }^{2} & R_{23}{ }^{2} & R_{23} R_{22} & R_{23} R_{21} & R_{22} R_{21} \\
R_{31}{ }^{2} & R_{32}{ }^{2} & R_{33}{ }^{2} & R_{33} R_{32} & R_{23} R_{31} & R_{32} R_{31} \\
2 R_{31} R_{21} & 2 R_{32} R_{22} & 2 R_{33} R_{23} & R_{33} R_{22}+R_{32} R_{23} & R_{33} R_{21}+R_{31} R_{23} & R_{31} R_{22}+R_{32} R_{21} \\
2 R_{31} R_{11} & 2 R_{32} R_{12} & 2 R_{33} R_{13} & R_{33} R_{12}+R_{32} R_{13} & R_{33} R_{11}+R_{31} R_{13} & R_{31} R_{12}+R_{32} R_{11} \\
2 R_{21} R_{11} & 2 R_{12} R_{22} & 2 R_{13} R_{23} & R_{13} R_{22}+R_{12} R_{23} & R_{13} R_{21}+R_{11} R_{23} & R_{11} R_{22}+R_{12} R_{21}
\end{array}\right)
$$
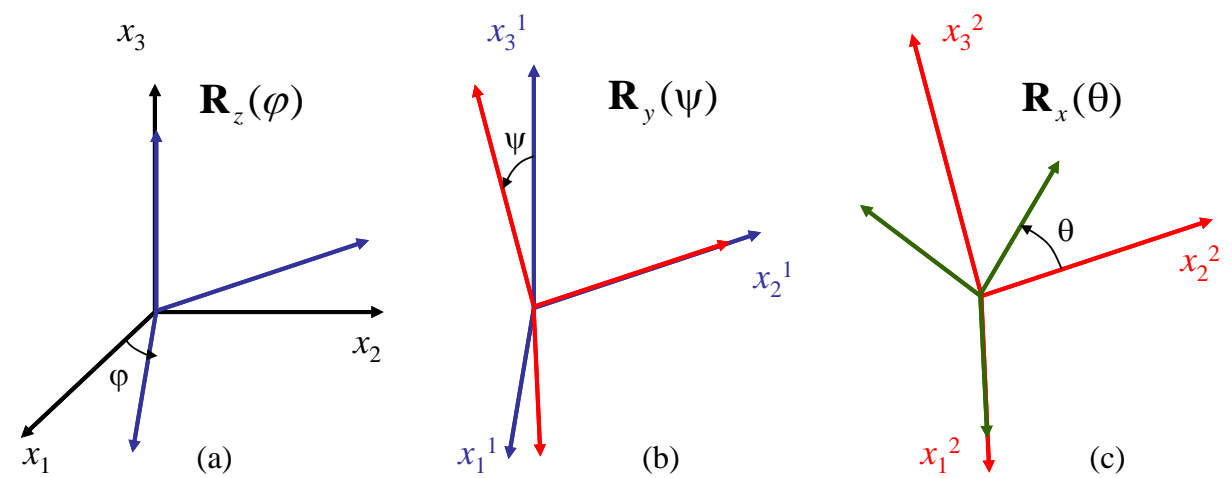

Figure 2 Three basic rotations: (a) rotation with respect to the $x_{3}$-axis, (b) rotation with respect to the $x_{2}{ }^{1}$-axis, (c) rotation with respect to the $x_{1}{ }^{3}$-axis, Dight and Dyskin (2007)

The transformation of the $6 \times 6$ compliance matrix $\mathbf{S}$ with the coordinate rotation is expressed as follows (Lekhnitskii, 1977):

$$
\mathbf{S}^{\prime}=\mathbf{Q S} \mathbf{Q}^{T}
$$

$$
\mathbf{Q}=\left(\begin{array}{cccccc}
R_{11}{ }^{2} & R_{12}{ }^{2} & R_{13}{ }^{2} & R_{12} R_{13} & R_{13} R_{11} & R_{12} R_{11} \\
R_{21}{ }^{2} & R_{22}{ }^{2} & R_{23}{ }^{2} & R_{23} R_{22} & R_{23} R_{21} & R_{22} R_{21} \\
R_{31}{ }^{2} & R_{32}{ }^{2} & R_{33}{ }^{2} & R_{33} R_{32} & R_{23} R_{31} & R_{32} R_{31} \\
2 R_{31} R_{21} & 2 R_{32} R_{22} & 2 R_{33} R_{23} & R_{33} R_{22}+R_{32} R_{23} & R_{33} R_{21}+R_{31} R_{23} & R_{31} R_{22}+R_{32} R_{21} \\
2 R_{31} R_{11} & 2 R_{32} R_{12} & 2 R_{33} R_{13} & R_{33} R_{12}+R_{32} R_{13} & R_{33} R_{11}+R_{31} R_{13} & R_{31} R_{12}+R_{32} R_{11} \\
2 R_{21} R_{11} & 2 R_{12} R_{22} & 2 R_{13} R_{23} & R_{13} R_{22}+R_{12} R_{23} & R_{13} R_{21}+R_{11} R_{23} & R_{11} R_{22}+R_{12} R_{21}
\end{array}\right)
$$

where $R_{i j}$ are the entries of the 3D rotation matrix $\mathbf{R}$ and the superscript ' $T$ ' stands for matrix transposition.

Given that the moduli determination in subcores can only be performed with limited accuracy, we try to find the compliances $\mathbf{S}$ in laboratory coordinate set, $\left(x_{1}, x_{2}, x_{3}\right)$ such that the results of subcore measurements are reproduced 'on average'. Thus assuming that the $x_{3}{ }^{(n)}$-axes of a local coordinate set coincides with the axis of subcore $(n)$, we chose $\mathbf{S}$ such that the components $S_{33}$ and $\left(S_{13}+S_{23}\right) / 2$ are made close to the values of $S_{\text {axial }}^{(n)}$ and $S_{\text {lateral }}^{(n)}$ in the sense of the least sum of squares, i.e. by solving the following minimisation problem:

$$
\sum_{n=1}^{N}\left[\left(\frac{S_{13}^{(n)}+S_{12}^{(n)}}{2}-S_{\text {lateral }}^{(n)}\right)^{2}+\left(S_{33}^{(n)}-S_{\text {axial }}^{(n)}\right)^{2}\right] \rightarrow \text { min, } \text { where } \quad \mathbf{S}^{(n)}=\mathbf{Q}^{(n)} \mathbf{S} \mathbf{Q}^{(n)^{T}}
$$


where $\mathbf{Q}^{(n)}$ is the rotation matrix that transfers the laboratory coordinate set to the coordinate set related to $n$-th subcore.

Since the compliances of $n$-th subcore linearly depend upon the unknown compliance matrix $\mathbf{S}$, the minimisation problem Equation (4) can be reduced to the problem of multiple linear regression by introducing the following 21 axillary (basis) matrices $\mathbf{B}^{\mathrm{km}}$ specified by their entries:

$$
B_{i j}^{k k}=\left\{\begin{array}{cc}
1 & \text { if } i=j=k \\
0 & \text { otherwise }
\end{array}, \quad B_{i j}^{k l}=\left\{\begin{array}{cc}
1 & \text { if } i=k, j=l \text { or } i=l, j=k \\
0 & \text { otherwise }
\end{array}\right.\right.
$$

In order to extract the components of matrix $\mathbf{S}$ in the regression analysis we introduce the following additional matrices:

$$
\mathbf{I}_{3}=\left(\begin{array}{l}
0 \\
0 \\
1 \\
0 \\
0 \\
0
\end{array}\right), \quad \mathbf{L}=\left(\begin{array}{llllll}
0 & 0 & 1 & 0 & 0 & 0 \\
0 & \frac{1}{2} & \frac{1}{2} & 0 & 0 & 0
\end{array}\right)
$$

subsequently,

$$
\mathbf{L S} \mathbf{I}_{3}=\left(\begin{array}{c}
S_{33} \\
\left(S_{13}+S_{23}\right) / 2
\end{array}\right)
$$

the least squares method Equation (4) can now be expressed in the following matrix form:

$$
\sum_{\substack{k, l=1 \\
k<l}}^{6}\left|\mathbf{L Q}^{(n)} \mathbf{B}^{k l} \mathbf{Q}^{(n)^{T}} \mathbf{I}_{3} S_{k l}-\mathbf{S}_{\exp }^{(n)}\right|^{2} \rightarrow \min , \quad \text { where } \quad \mathbf{S}_{\exp }^{(n)}=\left(\begin{array}{c}
S_{\text {axial }}^{(n)} \\
S_{\text {lateral }}^{(n)}
\end{array}\right), \quad n=1, \mathrm{~K}, N
$$

where |.| denotes the vector length.

Consider the simplest anisotropic case - the transverse-isotropy with the isotropy plane coinciding with the foliation plane. Let $\mathbf{Q}^{(n)}$ be the rotation matrix that transfers the laboratory coordinate set to the coordinate set related to $n$-th subcore, and $\mathbf{Q}^{f o l}$ be the rotation matrix that rotates the laboratory set in such a way that its $z$-axis becomes normal to the foliation plane.

With respect to the coordinate set associated with the foliation plane, the compliance matrix can be expressed through the transverse-isotropic moduli (so-called engineering constants) in the form of the following symmetric matrix (Lekhnitskii, 1977).

$$
\mathbf{S}=\left(\begin{array}{cccccc}
\frac{1}{E} & -\frac{v}{E} & -\frac{v^{\prime}}{E^{\prime}} & 0 & 0 & 0 \\
& \frac{1}{E} & -\frac{v^{\prime}}{E^{\prime}} & 0 & 0 & 0 \\
* & \frac{1}{E^{\prime}} & 0 & 0 & 0 \\
& & \frac{1}{2 G^{\prime}} & 0 & 0 \\
& & & \frac{1}{2 G^{\prime}} & 0 \\
& & & & \frac{1+v}{E}
\end{array}\right)
$$


where $E, v$ are the Young's modulus and Poisson's ratio respectively, corresponding to the loads in the foliation plane; and, $E^{\prime}, v^{\prime}$ and $G^{\prime}$ are the Young's modulus, Poisson's ratio and the shear modulus respectively, corresponding to the loads normal to the foliation plane.

For this particular case, it is convenient to use the single-index notation for the basis matrices Equation (5):

$$
\begin{aligned}
& \sum_{k=1}^{5}\left|\mathbf{B}_{\mathrm{k}}^{(n)} S_{k}-\mathbf{S}_{\exp }^{(n)}\right|^{2} \rightarrow \min \\
& \text { where } \quad \mathbf{B}_{k}^{(n)}=\mathbf{L} \mathbf{Q}^{(n)} \mathbf{Q}^{f o l^{-1}} \mathbf{B}^{k}\left(\mathbf{Q}^{(n)} \mathbf{Q}^{f o l^{-1}}\right)^{T} \mathbf{I}_{3}, \quad \mathbf{S}_{\exp }^{(n)}=\left(\begin{array}{c}
S_{\text {axial }}^{(n)} \\
S_{\text {lateral }}^{(n)}
\end{array}\right), \quad n=1, \mathrm{~K}, N
\end{aligned}
$$

The least squares criterion Equation (8) can now be expressed as:

$$
\begin{aligned}
& \sum_{k=1}^{5}\left|\mathbf{B}_{\mathrm{k}}^{(n)} S_{k}-\mathbf{S}_{\mathrm{exp}}^{(n)}\right|^{2} \rightarrow \min \\
& \text { where } \quad \mathbf{B}_{k}^{(n)}=\mathbf{L} \mathbf{Q}^{(n)} \mathbf{Q}^{f o l^{-1}} \mathbf{B}^{k}\left(\mathbf{Q}^{(n)} \mathbf{Q}^{f o l^{-1}}\right)^{T} \mathbf{I}_{3}, \quad \mathbf{S}_{\exp }^{(n)}=\left(\begin{array}{c}
S_{\text {axial }}^{(n)} \\
S_{\text {lateral }}^{(n)}
\end{array}\right), \quad n=1, \mathrm{~K}, N
\end{aligned}
$$

Here the basic compliances are $S_{1}=S_{11}, S_{2}=S_{33}, S_{3}=S_{12}, S_{4}=S_{13}, S_{5}=S_{44}$.

Equation (11) is solved by introducing a matrix $\mathbf{X}$ consisting of $2 N$ rows and five columns, the $k$-th column being formed by concatenation of $N$ vectors $\mathbf{B}_{k}^{(n)}$. Then the vector of the basic compliances $\mathbf{S}^{b}=\left(S_{k}\right), k=1, \mathrm{~K}, 5$ can be obtained as (Hakala et al., 2007).

$$
\mathbf{S}^{b}=\left(\mathbf{X}^{T} \mathbf{X}\right)^{-1} \mathbf{X}^{T} \mathbf{Y}
$$

where the column-vector of measurements $\mathbf{Y}$ consists of $2 N$ rows formed by concatenation of $N$ vectors $\mathbf{S}_{\text {exp }}^{(n)}$.

We use this method to reconstruct moduli for two types of rock, the first, designated 'A', being clearly anisotropic; the second, designated 'I', being nearly isotropic.

\subsection{Rock characterisation}

Both rocks tested came from cuperiferous deposits. The geological setting of the orebody for Rock A is as follows:

There are three major geological units comprising the footwall rhyodacite and breccias that are variably sericitically and chloritically altered the suphides which are polymetallic, massive cuperiferous, cuperiferous stockwork and barren pyrite, and the hangingwall volcanogenic sedimentary unit consisting of lavas, tuffs and argillites which are varuously hematitically and chloritic/sericitically altered.

The dominant foliation dips steeply to the north.

In addition, there are several large faults that dip steeply to the east intersecting the orebody. These fault's off-set the orebody to varying degrees at various distances along the strike of the orebody.

A typical intersection of the orebody is shown Figure 3 and a cross section of the orebody is shown in Figure 4. 


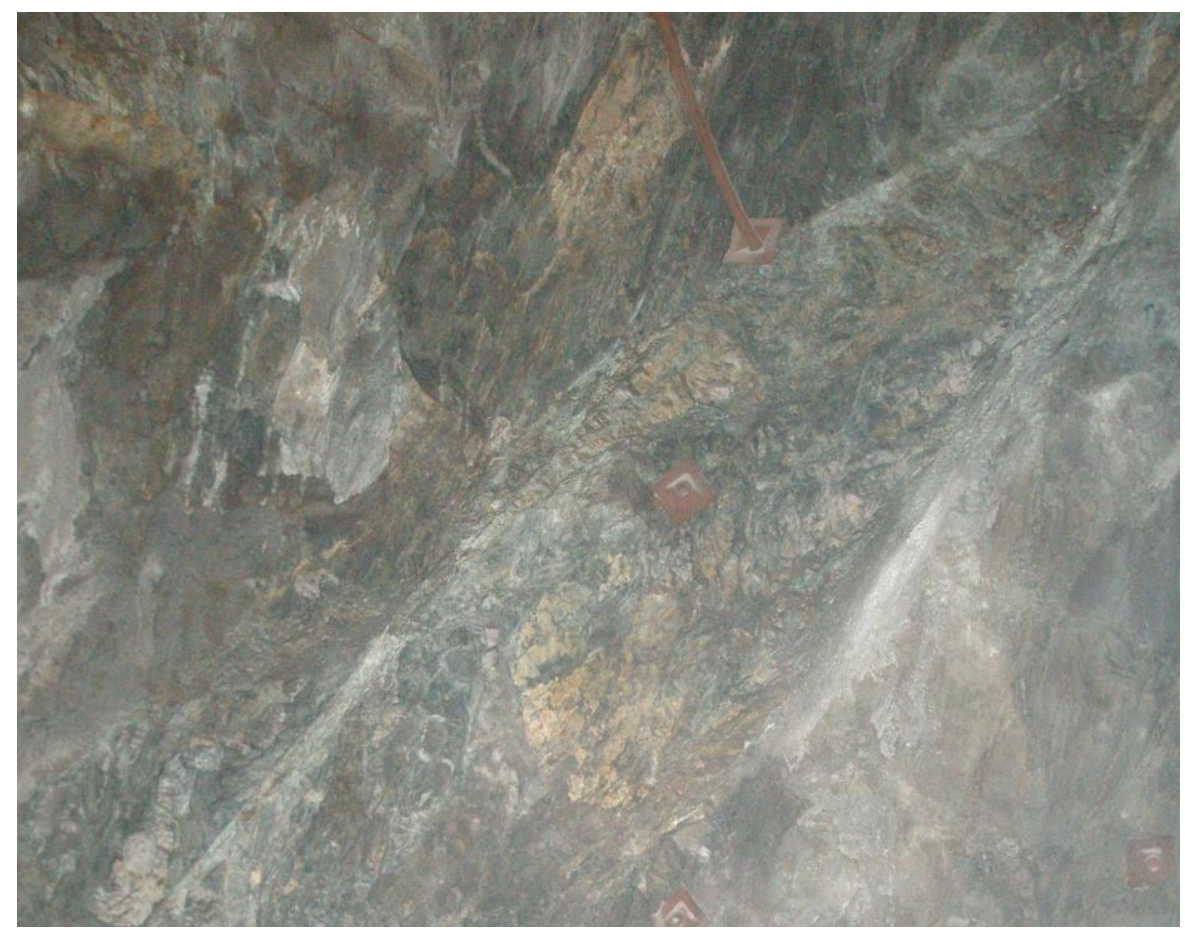

Figure 3 Photograph taken approaching the hangingwall contact in the 810 crosscut

The massive sulphide orebody is approximately $100-120 \mathrm{~m}$ wide, the contact to the undifferentiated volcanic sediments (UVS) dips gently to the north and plunges gently to the west.

For Rock I the geological setting is a massive cuperiferous porphyry deposit which is variable potassic, sericitic and chloritically altered hosted in a volanco-sedimentary host rock. The deposit is located on the plate boundary between India and Asia, but is clearly tectonically volcanic of origin. There is no preferred fabric in the orebody.

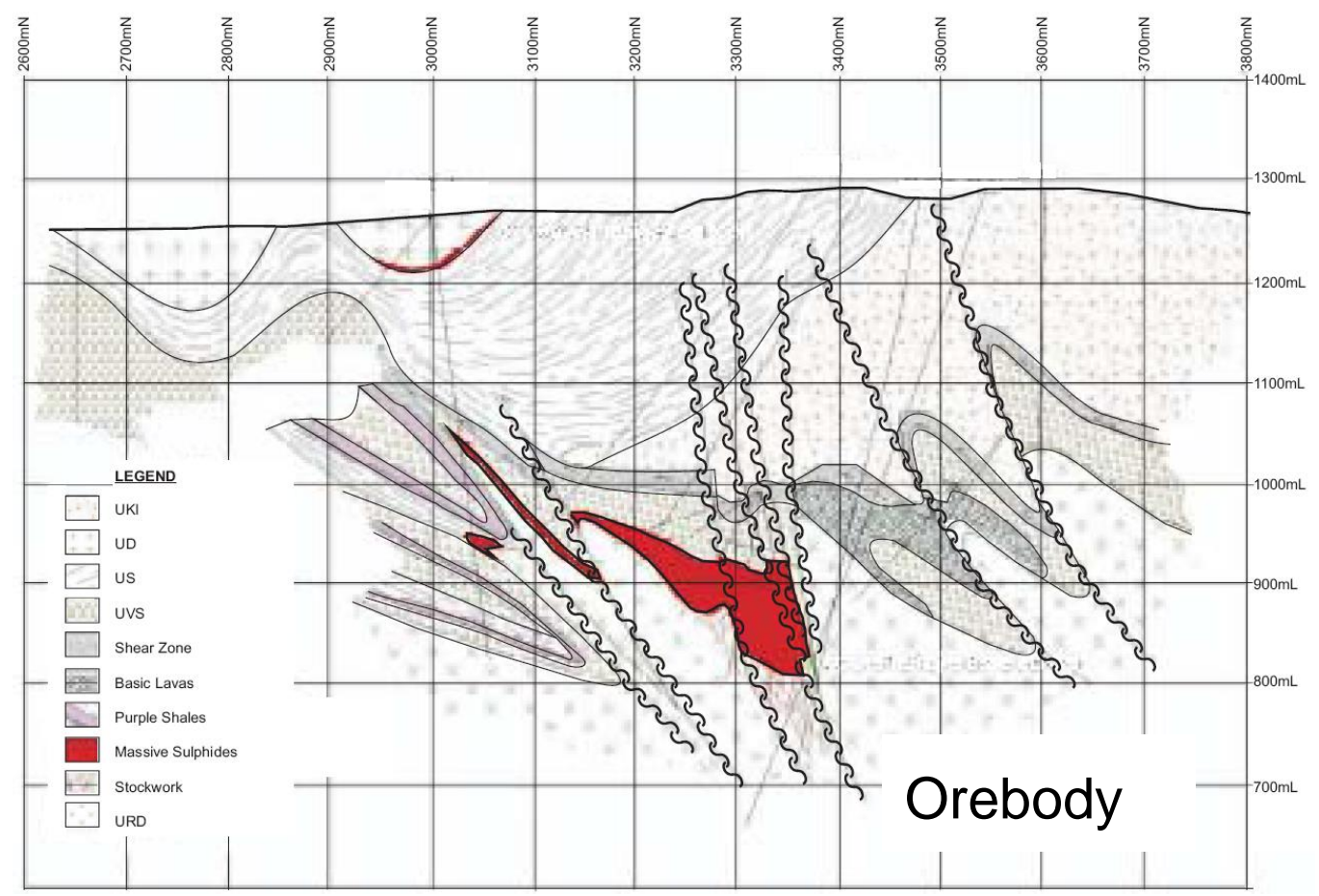

Figure 4 Typical cross section of the orebody 


\subsection{Results of moduli measurements in subcores}

The subcores were drilled at different directions specified by absolute azimuth (trend) and plunge which mark their real orientation in the rock mass. The orientations are shown in Tables 1 and 2. In some cases several subcores were drilled in the same direction. The subcores were subjected to displacement-controlled uniaxial compression. The strains were measured by four pairs of strain gauges. The axial strain measurements were conducted by two pairs of strain gauges axially glued to the opposite sides of the subcore. The positions of the pairs are marked as 0-180, and 90-270 referring to the angles (in degrees) along the circumference of the subcore from a certain reference point. Unfortunately, this reference point cannot be related to the true position in the rock mass; this information is currently unavailable. The subcores were not oriented as opposite to the cores which were. Two other pairs of strain gauges positioned on the same sides of the subcore measured the lateral strains.

From the stress-strain curves obtained, by averaging the readings in each pair of opposite gauges, the Young's modulus and Poisson's ratio were inferred. The results for both rocks are shown in Tables 1 and 2. The last two columns in each table show the inferred average axial and lateral compliances.

Table 1 Results of compliance determination in Rock A

\begin{tabular}{|c|c|c|c|c|c|c|c|c|}
\hline \multirow[b]{2}{*}{ Subcore } & \multirow{2}{*}{$\begin{array}{l}\text { Azimuth/ } \\
\text { Trend, } \\
\text { Deg }\end{array}$} & \multirow[b]{2}{*}{$\begin{array}{l}\text { Plunge, } \\
\text { Deg }\end{array}$} & \multirow[b]{2}{*}{$\begin{array}{c}E 0-180 \\
\mathrm{MPa}\end{array}$} & \multirow{2}{*}{\multicolumn{2}{|c|}{$\begin{array}{c}E 90-270 \\
\mathrm{MPa}\end{array}$}} & \multirow[b]{2}{*}{ v 90-270 } & \multicolumn{2}{|c|}{ Average Compliance } \\
\hline & & & & & & & $\begin{array}{c}S_{\text {axial }} \\
\mathrm{MPa}^{-1}\end{array}$ & $\begin{array}{l}S_{\text {lateral }} \\
\mathrm{MPa}^{-1}\end{array}$ \\
\hline \multirow[t]{2}{*}{$\mathrm{a}$} & 84 & 88 & 105.2 & 0.155 & 101.1 & 0.151 & 0.0097 & 0.00148 \\
\hline & & & 115.8 & 0.099 & 81.3 & 0.127 & 0.0105 & 0.00121 \\
\hline \multirow[t]{2}{*}{$\mathrm{b}$} & 174 & 0 & 146 & 0.34 & 145.3 & 0.108 & 0.0069 & 0.00154 \\
\hline & & & 119.4 & 0.136 & 123.2 & 0.184 & 0.0082 & 0.00132 \\
\hline \multirow[t]{4}{*}{$\mathrm{c}$} & 264 & 2 & 65.3 & 0.087 & 67.7 & 0.05 & 0.0150 & 0.00104 \\
\hline & & & 71 & 0.069 & 68.9 & 0.028 & 0.0143 & 0.00069 \\
\hline & & & 32.8 & 0.004 & 63.9 & 0.07 & 0.0231 & 0.00061 \\
\hline & & & 34.4 & 0.055 & 74.4 & 0.116 & 0.0213 & 0.00158 \\
\hline \multirow[t]{3}{*}{ d } & 309 & 1 & 74.3 & 0.128 & 31.3 & 0.388 & 0.0227 & 0.00706 \\
\hline & & & 82 & 0.132 & 37.4 & 0.237 & 0.0195 & 0.00397 \\
\hline & & & 87.1 & 0.133 & 115.2 & 0.231 & 0.0101 & 0.00177 \\
\hline \multirow[t]{2}{*}{$\mathrm{e}$} & 172 & 45 & 195 & 0.197 & 109.6 & 0.159 & 0.0071 & 0.00123 \\
\hline & & & 120.1 & 0.142 & 108.9 & 0.127 & 0.0088 & 0.00117 \\
\hline \multirow[t]{2}{*}{$\mathrm{f}$} & 264 & 47 & 110.8 & 0.097 & 114.8 & 0.126 & 0.0089 & 0.00099 \\
\hline & & & 157.7 & 0.169 & 151 & 0.179 & 0.0065 & 0.00113 \\
\hline
\end{tabular}


Table 2 Results of compliance determination in Rock I

\begin{tabular}{|c|c|c|c|c|c|c|c|c|}
\hline \multirow[b]{2}{*}{ Subcore } & \multirow{2}{*}{$\begin{array}{l}\text { Azimuth/ } \\
\text { Trend, } \\
\text { Deg }\end{array}$} & \multirow[b]{2}{*}{$\begin{array}{l}\text { Plunge, } \\
\text { Deg }\end{array}$} & \multirow[b]{2}{*}{$\begin{array}{c}E 0-180 \\
\mathrm{MPa}\end{array}$} & \multirow{2}{*}{\multicolumn{2}{|c|}{$\begin{array}{c}E 90-270 \\
\mathrm{MPa}\end{array}$}} & \multirow[b]{2}{*}{ v 90-270 } & \multicolumn{2}{|c|}{ Average Compliance } \\
\hline & & & & & & & $\begin{array}{c}S_{\text {axial }} \\
\mathrm{MPa}^{-1}\end{array}$ & $\begin{array}{l}S_{\text {lateral }} \\
\mathrm{MPa}^{-1}\end{array}$ \\
\hline \multirow[t]{3}{*}{$\mathrm{a}$} & 323 & 53 & 68.2 & 0.208 & 68.6 & 0.25 & 0.0146 & 0.00335 \\
\hline & & & 56.5 & 0.2 & 74.6 & 0.26 & 0.0156 & 0.00351 \\
\hline & & & 72 & 0.27 & 64.7 & 0.25 & 0.0147 & 0.00381 \\
\hline \multirow[t]{3}{*}{$\mathrm{b}$} & 143 & 37 & 67.7 & 0.26 & 75.2 & 0.3 & 0.0140 & 0.00391 \\
\hline & & & 72.6 & 0.25 & 72.6 & 0.27 & 0.0138 & 0.00358 \\
\hline & & & 70.5 & 0.23 & 73.5 & 0.24 & 0.0139 & 0.00326 \\
\hline \multirow[t]{3}{*}{$\mathrm{c}$} & 53 & 0 & 65 & 0.26 & 70.6 & 0.25 & 0.0148 & 0.00377 \\
\hline & & & 65 & 0.26 & 70.6 & 0.25 & 0.0148 & 0.00377 \\
\hline & & & 73.8 & 0.22 & 74.8 & 0.31 & 0.0135 & 0.00356 \\
\hline \multirow[t]{2}{*}{$\mathrm{d}$} & 92 & 25 & 101.4 & 0.3 & 85.4 & 0.28 & 0.0108 & 0.00312 \\
\hline & & & 72.5 & 0.17 & 71.6 & 0.24 & 0.0139 & 0.00285 \\
\hline $\mathrm{e}$ & 143 & 82 & 79.1 & 0.28 & 75.6 & 0.25 & 0.0129 & 0.00342 \\
\hline \multirow[t]{3}{*}{$\mathrm{f}$} & 264 & 34 & 82.4 & 0.28 & 73.7 & 0.25 & 0.0129 & 0.00340 \\
\hline & & & 70.4 & 0.24 & 73.9 & 0.25 & 0.0139 & 0.00340 \\
\hline & & & 76.5 & 0.26 & 67.2 & 0.28 & 0.0140 & 0.00378 \\
\hline
\end{tabular}

It is seen from the Table 1 that Rock A shows quite different values of the moduli in different directions. This is characteristic of considerable anisotropy, which justifies the designation ' $A$ '. It is interesting that the moduli exhibit considerable variations even for the same directions. This phenomenon is probably due to the fact that some subcores intersect larger scale elements of rock structure, like joist or foliation planes, while others do not. We will analyse this hypothesis in the following section. Opposite to it, for Rock I, Table 2, the moduli do not change much with the direction which suggests that the rock is approximately isotropic and hence designated ' $I$ '.

We reconstruct the moduli using the algorithm detailed in Section 2.1. For Rock A we assumed that it is transverse isotropic with the plane of isotropy coinciding with the foliation plane. The latter was oriented $\mathrm{N}-\mathrm{S}$ with the plunge of $75^{\circ}$. The reconstruction gives:

$$
\begin{array}{ll}
E_{\text {parallel foliation }}=69 \mathrm{GPa} & E_{\text {normal foliation }}=153 \mathrm{GPa} \\
v_{\text {parallel foliation }}=0.186 & v_{\text {normal foliation }}=0.167 \\
G_{\text {parallel foliation }}=29 \mathrm{GPa} & G_{\text {normal foliation }}=30 \mathrm{GPa}
\end{array}
$$

Sensitivity analysis performed by randomly (and independently) varying measurements within $\pm 10 \%$ shows the following relative variations (in \%) of the reconstructed moduli:

$$
\begin{aligned}
& \delta E_{\text {parallel foliation }}=2.7 \\
& \delta E_{\text {normal foliation }}=6 \\
& \delta v_{\text {parallel foliation }}=7.7 \\
& \delta v_{\text {normal foliation }}=16.7 \\
& \delta G_{\text {normal foliation }}=5
\end{aligned}
$$


It is seen that the variations are of the same order as the induced disturbance in the input data which suggests that the reconstruction results are stable.

A similar procedure for Rock I assuming again transverse isotropy with the plane of isotropy oriented the same way as for Rock A gives:

$$
\begin{array}{ll}
E_{\text {parallel foliation }}=60 \mathrm{GPa} & E_{\text {normal foliation }}=68 \mathrm{GPa} \\
v_{\text {parallel foliation }}=0.12 & v_{\text {normal foliation }}=0.38 \\
G_{\text {parallel foliation }}=27 \mathrm{GPa} & G_{\text {normal foliation }}=27 \mathrm{GPa}
\end{array}
$$

Similarly, the sensitivity analysis gives:

$$
\begin{aligned}
& \delta E_{\text {parallel foliation }}=3.248 \\
& \delta E_{\text {normal foliation }}=4.483 \\
& \delta v_{\text {parallel foliation }}=25.6 \\
& \delta v_{\text {normal foliation }}=4.62 \\
& \delta G_{\text {normal foliation }}=2.957
\end{aligned}
$$

It is seen that the difference between the moduli in different directions is relatively small, except the Poisson's ratios. It should be noted though that the sensitivity analysis shows high sensitivity of one of the Poisson's ratios which suggests reduced accuracy in its reconstruction. Given this Rock I can indeed be assumed isotropic.

\section{A mechanism of variability}

We now turn our attention to the nature of high variability of the moduli measured on different subcores with the same orientation. We note that it is a specific feature of the anisotropic rock; the isotropic rock shows quite consistent measurements for subcores of the same orientation.

From the theory of elasticity, the anisotropy is a property associated with orientation rather than the heterogeneity, therefore anisotropy alone is insufficient to explain the observed variability. One however notes that the properties of rocks, including the elastic properties are scale dependent: what is seen as heterogeneous rock at a small scale can be perfectly described by homogeneous elasticity at larger scales. Similarly, what behaves as homogeneous elastic material at the scale of cores and stress measurements can be heterogeneous at the scale of small subcores. As an example relevant to this study consider an isotropic rock transversed by a set of parallel joints characterised by spacing $d$ and the normal $k_{n}$ and shear $k_{s}$ stiffnesses, Figure 1. If one considers such fractured rock at a large scale, that is the scale much larger than the joint spacing, the deformation can be described in terms of a homogeneous equivalent material which is in this case transverse isotropic with the plane of isotropy parallel to the joints.

In order to analyse this concept we introduce a coordinate frame $\left(x_{1}, x_{2}, x_{3}\right)$ with the $x_{3}$-axis directed normal to the joints. Then the compliances of the homogeneous equivalent material (so-called effective compliances) can be expressed as follows (Jager et al., 2007):

$$
\mathbf{S}=\left(\begin{array}{cccccc}
\frac{1}{E_{0}} & -\frac{v_{0}}{E_{0}} & -\frac{v_{0}}{E_{0}} & 0 & 0 & 0 \\
-\frac{v_{0}}{E_{0}} & \frac{1}{E_{0}} & -\frac{v_{0}}{E_{0}} & 0 & 0 & 0 \\
-\frac{v_{0}}{E_{0}} & -\frac{v_{0}}{E_{0}} & \frac{1}{E_{0}}+\frac{1}{k_{n} d} & 0 & 0 & 0 \\
0 & 0 & 0 & \frac{1}{2}\left(\frac{1}{G_{0}}+\frac{1}{k_{s} d}\right) & 0 & 0 \\
0 & 0 & 0 & 0 & \frac{1}{2}\left(\frac{1}{G_{0}}+\frac{1}{k_{s} d}\right) & 0 \\
0 & 0 & 0 & 0 & 0 & \frac{1}{2 G_{0}}
\end{array}\right)
$$


where $E_{0}, v_{0}$ and $G_{0}=E_{0} / 2\left(1+v_{0}\right)$ are the moduli of the intact (isotropic) rock. We can see that in this linear approximation (interaction of the neighbouring joints is neglected) only compliances $S_{33}$ and $S_{44}, S_{55}$ are increased by $1 / k_{n} d$ and $1 / 2 k_{s} d$ respectively.

Consider now a short subcore, with the length smaller than the joint spacing, Figure 1. As its position with respect to the joints is arbitrarily, in some cases it will be intersected by a joint, while in other it will not. The probability that the subcore is intersected by a joint can be easily found if the subcore diameter is neglected. This probability is:

$$
P=\frac{l}{d} \sin \alpha
$$

where $\alpha$ is the angle of inclination of the subcore axis to the plane of joints.

If no intersection occurs (probability $1-P$ ) then the subcore is isotropic with moduli $E_{0}, v_{0}$ and $G_{0}$ and the compliance matrix:

$$
\mathbf{S}_{1-P}=\left(\begin{array}{cccccc}
\frac{1}{E_{0}} & -\frac{v_{0}}{E_{0}} & -\frac{v_{0}}{E_{0}} & 0 & 0 & 0 \\
-\frac{v_{0}}{E_{0}} & \frac{1}{E_{0}} & -\frac{v_{0}}{E_{0}} & 0 & 0 & 0 \\
-\frac{v_{0}}{E_{0}} & -\frac{v_{0}}{E_{0}} & \frac{1}{E_{0}} & 0 & 0 & 0 \\
0 & 0 & 0 & \frac{1}{2 G_{0}} & 0 & 0 \\
0 & 0 & 0 & 0 & \frac{1}{2 G_{0}} & 0 \\
0 & 0 & 0 & 0 & 0 & \frac{1}{2 G_{0}}
\end{array}\right)
$$

If the intersection occurs (probability $P$ ) then the effective compliances relative to the coordinate frame $\left(x_{1}, x_{2}, x_{3}\right)$ are:

$$
\mathbf{S}_{P}=\left(\begin{array}{cccccc}
\frac{1}{E_{0}} & -\frac{v_{0}}{E_{0}} & -\frac{v_{0}}{E_{0}} & 0 & 0 & 0 \\
-\frac{v_{0}}{E_{0}} & \frac{1}{E_{0}} & -\frac{v_{0}}{E_{0}} & 0 & 0 & 0 \\
-\frac{v_{0}}{E_{0}} & -\frac{v_{0}}{E_{0}} & \frac{1}{E_{0}}+\frac{1}{k_{n} l \sin \alpha} & 0 & 0 & 0 \\
0 & 0 & 0 & \frac{1}{2}\left(\frac{1}{G_{0}}+\frac{1}{k_{s} l \sin \alpha}\right) & 0 & 0 \\
0 & 0 & 0 & 0 & \frac{1}{2}\left(\frac{1}{G_{0}}+\frac{1}{k_{s} l \sin \alpha}\right) & 0 \\
0 & 0 & 0 & 0 & 0 & \frac{1}{2 G_{0}}
\end{array}\right)
$$

Subsequently, the average compliance is:

$$
\langle\mathbf{S}\rangle=P \mathbf{S}_{P}+(1-P) \mathbf{S}_{1-P}
$$

Substituting Equations (14) to (16) into Equation (17) and comparing the result with Equation (13) one finally obtains:

$$
\langle\mathbf{S}\rangle=\mathbf{S}
$$


Thus, simple averaging of the measurements of subcores or, equivalently, applying the least square method procedure described in Section 2.1 one can obtain the large-scale moduli needed for the interpretation of the results of the stress relieve measurements. However, the variance (square of standard deviation) of, for instance, $S_{33}$ is:

$$
\operatorname{Var}\left(S_{33}\right)=\left\langle\left(S_{33}-\left\langle S_{33}\right\rangle\right)^{2}\right\rangle=\frac{1}{k_{n}^{2} d}\left[\frac{1}{l \sin \alpha}-\frac{1}{d}\right] \underset{\alpha \rightarrow 0}{\longrightarrow} \infty
$$

Thus there are instances when the variance and hence the standard deviation can be very large which may serve as an explanation for the high variability of the moduli in Rock A.

\section{Conclusions}

The stress measurements based on the stress relief methods can be influenced by rock anisotropy. The determination of anisotropic moduli requires considerable amount of testing in a variety of directions. A method based on testing subcores drilled out in different directions may suffer from high variations of the moduli measured in subcores even if they were cut in the same directions. This happens when the anisotropy is an effective property seen at the scale larger than the subcore length. A possible scenario is the anisotropy induced by parallel joints with the spacing exceeding the subcore length. In this case, the result of the moduli determination in a particular subcore is controlled by whether the subcore happens to intersect a joint or not. The average moduli (over many subcores) will still be representative of the large-scale effective moduli, however the standard deviation has a potential of becoming very large leading to the observed variability in the subcore moduli.

\section{Acknowledgements}

We acknowledge the assistance from Sudeep Pant, Coffey Mining Pty Ltd in the initial treatment of experimental data. Professor Dyskin acknowledges the financial support from the Australian Research Council through the Discovery Grant DP0771044.

\section{References}

Amadei, B. (1983) Lecture notes in engineering - rock anisotropy and theory of stress measurements. C.A. Brebbia, S.A. Orszag (editors), Berlin, Springer, pp. 233-241.

Amadei, B. and Goodman, R.E. (1982) The Influence of Rock Anisotropy on Stress Measurements by Overcoring Techniques, Rock Mechanics, 15, pp. 167-180.

Borsetto, M., Martinetti, S. and Ribacchi, R. (1984) Interpretation of in situ stress measurements in anisotropic rocks with the doorstopper method. Rock Mechanics and Rock Engineering, 17, pp. 167-182.

Dight, P.M. and Dyskin, A.V. (2007) Accounting for the effect of rock mass anisotropy in stress measurements. Deep Mining 07, Proceedings 4th International Seminar on Deep and High Stress Mining, Y. Potvin (editor), Australian Centre for Geomechanics, pp. 415-424.

Goodman, R.E. (1963) Subaudible noise during compression of rocks. Bull. Geol. Soc. Am. 74, pp. 487-490.

Hakala, M., Kuula, H. and Hudson, J.A. (2007) Estimating the transversely isotropic elastic intact properties for in-situ stress measurement data reduction: A case study of the Olkiluoto mica gneiss, Finland. Int. Jnl. Rock Mechanics and Mining Sciences, 44, pp. 14-46.

Jaeger, J.C., Cook, N.G.W. and Zimmerman, R.W. (2007) Fundamentals of Rock Mechanics. Backwell Publ., 475 p.

Kurita, K. and Fujii, N. (1979) Stress memory of crystalline rocks in acoustic emission. Geophys. Res. Lett. 6, pp. 9-12.

Lavrov, A. (2002) The Kaiser effect in rocks: principles and stress estimation techniques. Int. Jnl. Rock Mech. Min. Sci., 40, pp. 151-171.

Lekhnitskii, S.G. (1977) Theory of Elasticity of an Anisotropic Body. Mir, Moscow, 415 p. 
